\title{
Treatment of primary cutaneous anaplastic large cell lymphoma
}

\author{
Bong Soo Baik', \\ Wu Seop Lee', \\ So Young Ji', \\ Ki Sung Park ${ }^{1}$, \\ Wan Suk Yang ${ }^{1}$, \\ Sun Young Kim² \\ Departments of ${ }^{1}$ Plastic and \\ Reconstructive Surgery and ${ }^{2}$ Pathology, \\ Dong-Kang General Hospital, Ulsan, \\ Korea
}

\begin{abstract}
Primary cutaneous anaplastic large cell lymphoma (C-ALCL) is a rare subtype of primary cutaneous lymphoma with a favorable prognosis. Primary cutaneous CD30+ lymphoproliferative disorders, which include C-ALCL and lymphomatoid papulosis, are the second most common group of cutaneous T-cell lymphomas. C-ALCL is comprised of large cells with anaplastic, pleomorphic, or immunoblastic cytomorphology, and indeed, more than $75 \%$ of the tumor cells express the CD30 antigen. C-ALCL clinically presents with solitary or localized reddish-brown nodules or tumors, and sometimes indurated papules, and they may be with ulceration covering with dark eschar. Multifocal lesions are seen in $20 \%$ of the patients. Extracutaneous dissemination, which mainly involves the regional lymph nodes, occurs in $10 \%$ of patients. A 69 -year-old man noticed a mild elevated cutaneous lesion containing central ulceration covering with brownish black necrotic tissue on the right lower lip, and the lesion was surgically removed. After the first operation, another skin lesion was developed and the histological examination confirmed the diagnosis, C-ALCL. Eight specimens were excised during the 7-month follow-up period. The patient started the treatment with low-dose oral methotrexate $(15 \mathrm{mg} / \mathrm{wk})$ and there was no recurrence for 11 months.
\end{abstract}

Keywords: Lymphoma, primary cutaneous anaplastic large cell / Lymphoma, T-cell, cutaneous

\section{INTRODUCTION}

Clinicians, who treat the skin ulcers and nodules, can be confronted with rare skin malignancy. We have experienced a patient with ulcerated nodule covering with brownish black eschar and the resected specimen was diagnosed as primary cutaneous anaplastic large cell lymphoma (C-ALCL) by various pathological procedures. C-ALCL is a rare type of skin lymphomas with a good prognosis. However, long-term follow-up is essential for this disease. The patient was treated with excision of the skin lesions and oral methotrexate medication.

\section{Correspondence: So Young Ji}

Department of Plastic and Reconstructive Surgery, Dong-Kang General Hospital, 239 Taehwa-ro, Jung-gu, Ulsan 44455, Korea

E-mail: csy0203@hanmail.net

Received October 1, 2018 / Revised February 20, 2019 / Accepted March 15, 2019

\section{CASE REPORT}

A 69-year-old man visited Dong-Kang General Hospital with a mildly elevated cutaneous lesion, and the central ulceration was covered with a brownish black necrotic tissue on the right lower lip. The necrotic tissue grew slowly to $1 \mathrm{~cm}$ in diameter for the last 2 months (Fig. 1A). The patient's past history was trivial. The lesion was excised under local anesthesia and the excised wound was sutured primarily. Histopathologic findings revealed that the skin ulcer with the necrotic tissue and chronic inflammation was mainly spread around the skin appendages. When he re-visited our hospital about 3 months after the first excision of the cutaneous lesion, he had two additional new nodules. One nodule was found on the right buttock. This diffusely bulging nodule had a central brownish black necrotic tissue which was clearly demarcated from the surrounding tissue 

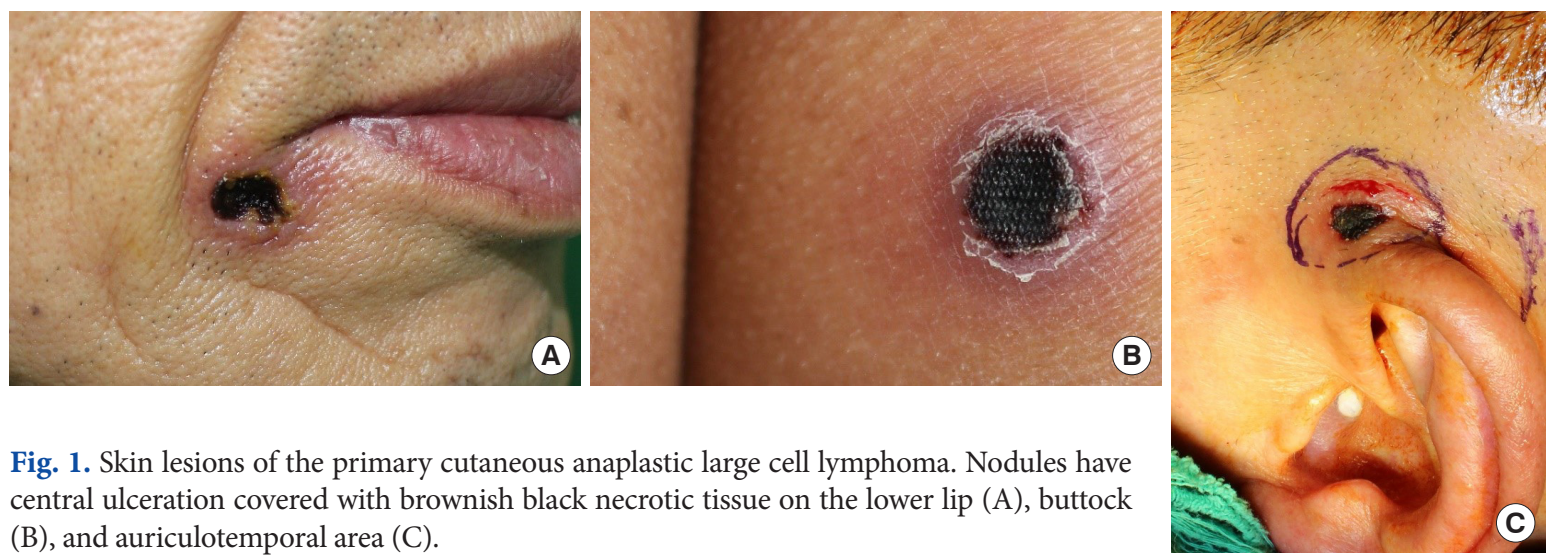

Fig. 1. Skin lesions of the primary cutaneous anaplastic large cell lymphoma. Nodules have central ulceration covered with brownish black necrotic tissue on the lower lip (A), buttock (B), and auriculotemporal area (C).
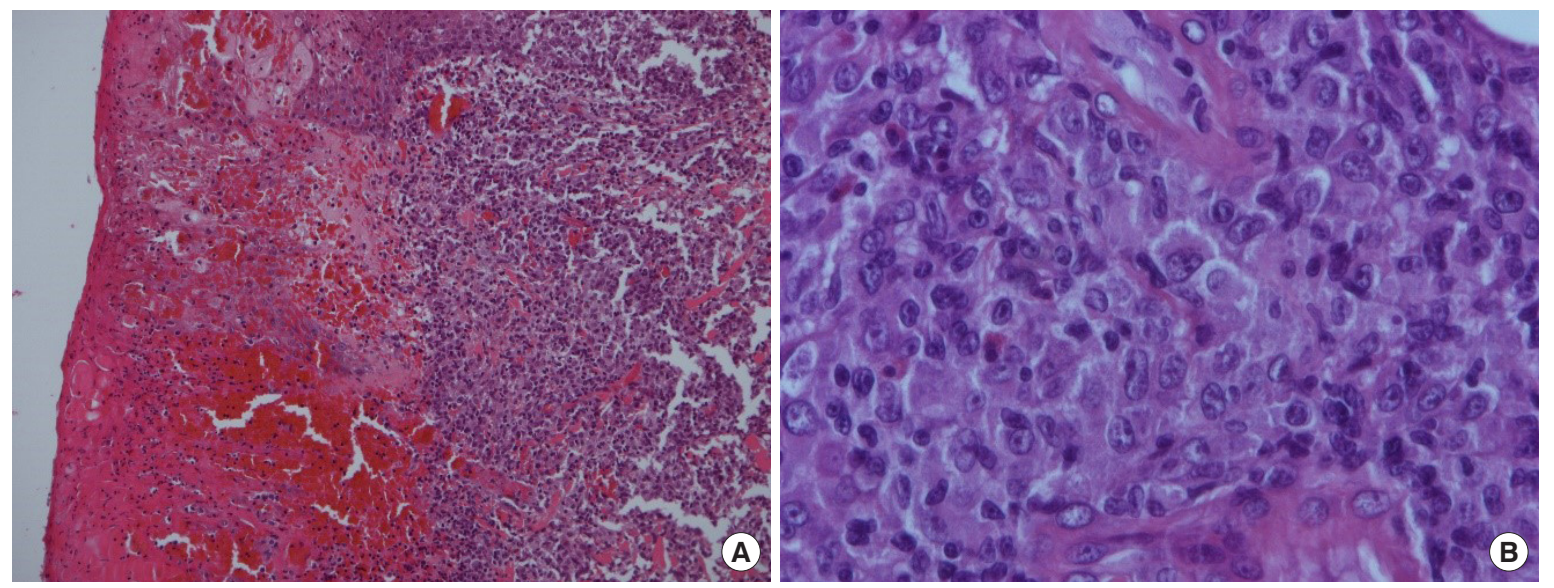

Fig. 2. Histopathological images. (A) Photomicrograph shows extensive infiltration of atypical lymphoid cells in the dermis with hemorrhagic necrosis $(\mathrm{H} \& \mathrm{E}, \times 100)$ and $(\mathrm{B})$ confluent sheets of medium-sized to large pleomorphic or anaplastic cells $(\mathrm{H} \& \mathrm{E}, \times 400)$.

(Fig. 1B). The other nodule was located on the left auriculotemporal area, and it also had the central necrotic tissue (Fig. 1C). Indeed, the buttock and auriculotemporal nodules were excised. The buttock wound has primarily closed. Nevertheless, the auriculotemporal wound was covered with a superiorly based retro-auricular flap because the wound was too wide to suture primarily. As a matter of fact, there was extensive hemorrhagic necrosis with ulcer and the infiltration of medium to large-sized pleomorphic and anaplastic cells with small lymphocytes in the dermis and subcutaneous tissues (Fig. 2). A followed exam revealed the tumor cells had clonally rearranged $\mathrm{T}$ cell receptor genes. The expression patterns of their surface markers were $\mathrm{CD} 3(+), \mathrm{CD} 5(+), \mathrm{CD} 20(-)$, CD56(-), CD30(+) $>75 \%$ in large atypical lymphoid cells (Fig. 3); CD4(-), CD8(-), Ki-67 labeling index $>80 \%$ (high proliferative activity) and Epstein-Barr virus in situ hybridization (-). Especially both epithelial membrane antigen (EMA) and anaplastic lymphoma kinase (ALK) translocation were negative: primary C-ALCLs in the skin are typically negative for the ALK; whereas, systemic C-ALCLs that secondarily involved the skin are ALK-1 reactive and

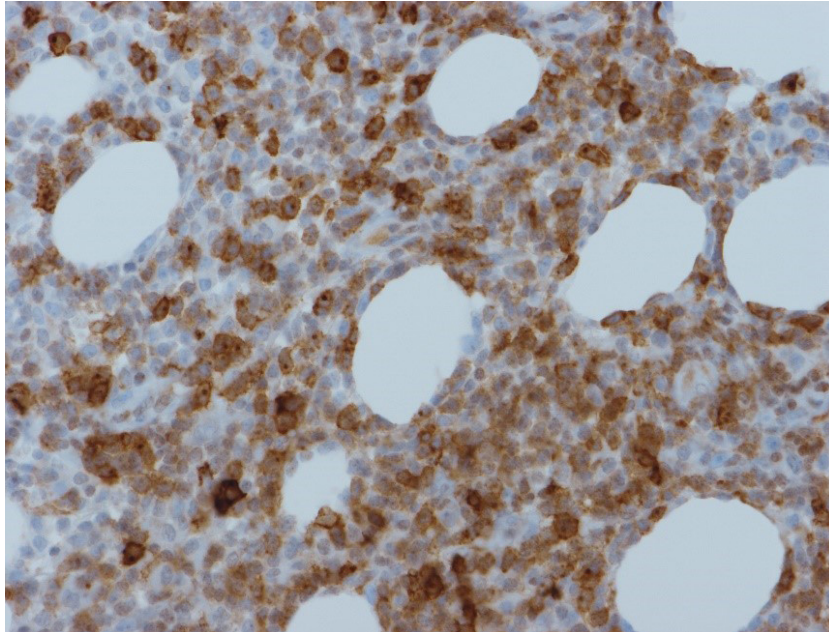

Fig. 3. Immunostaining for CD30 is positive in atypical cells $(\times 200)$.

express EMA. Concerning with the clinical features such as the multiple lesions and the patient's age (69 years old), they were finally diagnosed as primary cutaneous CD30+ T-cell lymphoproliferative disorders (LPD), and indeed consistent with the 

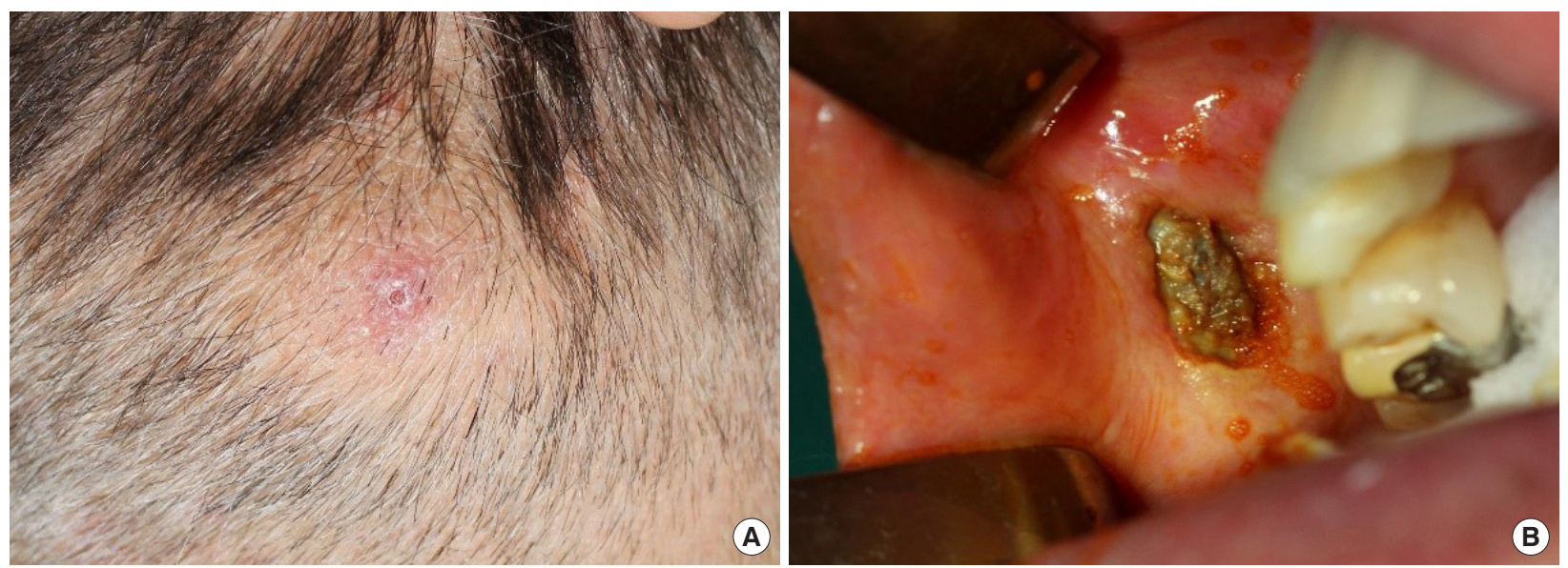

Fig. 4. (A) A small papule on the occipital scalp. (B) A brownish necrotic tissue on the right oral mucosa.

clinical manifestations of primary C-ALCL. In positron emission tomography-computed tomography, no signs of lymph node involvement and metastasis were found, and therefore, we staged him as IB (T2aNOM0).

Two months after the excision of the buttock and auriculotemporal nodules, a new elevated papule with redness of surrounding skin was found on the occipital area (Fig. 4A). The excised specimen was confirmed as primary cutaneous CD30 positive LPD. He was referred to another hospital with our data and biopsy specimen. In that hospital, further evaluations were performed. Bone marrow study showed no definite evidence of bone marrow involvement of lymphoma and immunohistochemistry results were $\mathrm{CD} 30(+), \mathrm{CD} 4(+), \mathrm{CD} 3(-), \mathrm{CD} 20(-)$, $\mathrm{CD} 8(-)$, and $\mathrm{ALK}(-)$. After a re-examination of his biopsy specimen, his final diagnosis was CD30 positive LPD.

The patient came back to our hospital with brownish necrotic tissue on the oral mucosal surface of the right cheek, a month after his visit to another hospital (Fig. 4B). Excision and biopsy were conducted on the necrotic tissue including surrounding mucosal tissue. The diagnosis was the same as one of the previous biopsies. After the result of the last biopsy on the oral mucosa, the patient has been treated with low-dose oral methotrexate once in a week $(15 \mathrm{mg} / \mathrm{wk})$. The wound of the oral mucosa was well healed. Most of all, there was no recurrence for the last 11 months after the first oral methotrexate medication.

\section{DISCUSSION}

Lymphomas are classified into Hodgkin's lymphoma (HL) and non-Hodgkin's lymphoma (NHL). The types of NHL are further differentiated into nodal and extranodal NHL. Next to the gastrointestinal tract, the skin is the second most common site of extranodal NHL. As a matter of fact, the head and neck are the most common site of presentation of skin lymphomas [1]. Primary cutaneous lymphoma arising in the skin is restricted to the skin at the time of diagnosis and staging, and it is often confined to the skin for a long period of time. One of the main features of secondary cutaneous lymphomas is a disseminated nodal infiltration or an extranodal lymphoma with the skin infiltration [2]. The WHO/EORTC (World Health Organization/ European Organization for Research and Treatment of Cancer) classification of primary cutaneous lymphomas includes three main categories based on the origin of cell: cutaneous T-cell lymphoma (CTCL), cutaneous B-cell lymphoma, and immature hematologic malignancies [1]. Primary CTCLs, the most common primary cutaneous lymphoma, mainly arise from a malignant population of lymphocyte in the skin [2]. CTCLs include mycosis fungoides (most common type), Sézary syndrome, CD30+ cutaneous LPD, primary cutaneous peripheral T-cell lymphoma, and adult T-cell leukemia/lymphoma $[1,2]$.

Primary cutaneous CD30+ LPD are the second most common group of CTCL, accounting for approximately $30 \%$ of CTCL cases. LPD is characterized by expression of the cell-surface receptor $\mathrm{CD} 30+$, which is a marker of activated $\mathrm{T}$ cells and a member of the tumor necrosis factor superfamily [3]. LPD include lymphomatoid papulosis (LYP) as a chronic recurrent lymphoproliferative skin disease and primary C-ALCL which is known for a low-grade malignancy lymphoma [3]. Histopathologically, these lesions are defined by the variable number of atypical CD30+ lymphocytes, and indeed, they are often mixed with granulocytes [4]. C-ALCL is a common type of CTCL, accounting for approximately $9 \%$ of all CTCLs with a median age of 60 years [4]. The majority of C-ALCL patients may present with solitary or localized (ulcerating) nodules or papules-the ulceration covering with necrotic tissue in our case.

Primary cutaneous lymphomas primarily arise on the skin 
without systemic involvement, but for many patients with skin lymphomas, the lymphomas will develop into extracutaneous diseases [1]. Primary cutaneous lymphomas represent approximately $19 \%$ of all extranodal NHL, and primary CTCLs constitute about $65 \%-71 \%$ of all primary cutaneous lymphomas [2,3]. The annual incidence of skin developing extranodal NHL was estimated to be 1:100,000 approximately. Indeed, it is the second most common group of NHL after the gastrointestinal tract [1]. As a matter of fact, the percentage of primary cutaneous CD30+ LPD in primary cutaneous lymphomas was approximately $20.05 \%$ in the United States [1]. Similarly, primary cutaneous CD30+ LPD constituted approximately 20.7\%, and CTCLs constituted approximately $88 \%$ of all primary cutaneous lymphoma in Korea [5].

Primary cutaneous CD30+ LPD include primary C-ALCL and LYP [1]. Differential diagnosis between C-ALCL and LYP is required clinicopathologically because these diseases have overlapping histopathologic, phenotypic, and genetic features. LYP occurs in adults with a median age of 45 years old, but CALCL with the median age of 60 years old [4]. Korean patients with the diagnoses of LYP and primary C-ALCL, however, were relatively young with median ages of 33 years old and 47 years old, respectively [5]. LYP manifests as a recurrent eruption of papules and nodules that are restricted to the skin, and it eventually self-resolves. Skin lesions disappear within 3 to 12 weeks and may leave scars. Histopathologically, the patterns of skin infiltration are in great variation. Therefore, the characteristic features should be mentioned in terms of epidermotropism, density, and even with or without the presence of large atypical cells. The depth of the skin infiltration for each case is also different, the epidermis to the subcutaneous layers [4]. LYP is characterized by multiple papulonodular lesions which undergo spontaneous regression after a few weeks or months. The disease usually persists for years or decades but has an excellent prognosis [2]. In contrast, C-ALCL patients often present with solitary or grouped, frequently ulcerated large tumors [2]. Histologically, the majority of them have round, oval, or irregularly shaped nuclei with prominent eosinophilic nucleoli and abundant cytoplasm; therefore, exhibiting characteristic morphology of anaplastic cells with a high mitotic rate [4].

The treatment of cutaneous lymphomas is either radiation or chemotherapy alone or two combined. Localized radiation therapy may be adequate for patients who have localized disease. Systemic treatment is indicated for the majority of patients who have extracutaneous lymphomas $[1,6]$. Some patients undergo surgical excision and biopsy as their only choice of treatment [7]. Different types of primary cutaneous lymphoma and different subtype of CTCLs, however, require different treat- ments. The overall 5-year relative survival rate for the patients with CTCLs is about $85 \%$, and the survival rates are similar between males and females with CTCLs (84\% and 88\%, respectively) [3]. Prognostic importance is probably the initial extent of the disease and histopathological nature of the lesion [2]. The prognosis of C-ALCL is favorable with a 5-year survival rate of $95 \%$, even in patients with involvement of regional lymph nodes [2]. Surgical excision and radiotherapy are first-line therapies in solitary tumors [1]. The use of low-dose methotrexate (5-20 mg/wk) is very effective for grouped or multiple lesions of C-ALCL [1]. Multi-agent chemotherapy should be applied exclusively to the patients with extracutaneous spread of lesions $[1,2]$.

Low-dose methotrexate is commonly used as a first-line treatment of LYP, not in C-ALCL. Only anecdotal cases of methotrexate treatment on C-ALCL are reported [8]. Although the administration of methotrexate in multifocal C-ALCL is not yet proved as useful with classified clinical trials, it seems to be acceptable by considering the general expert's experience $[6,8]$.

Hepatotoxicity, pulmonary toxicity, and myelosuppression are known as the adverse effects of low-dose methotrexate therapy [9-12], It is also known to cause a rash, fever, alopecia, and gastrointestinal problems such as nausea and stomatitis. For this reason, tapering the dose of methotrexate is very important to minimize the side effects. Unfortunately, the ideal methotrexate-taper protocol has not been established yet.

As to the record of low-dose methotrexate use in solitary CALCL, at least 4 months are required to declare complete remission [8]. In this case, we declared the complete remission in September; indeed, 4 months after the 1st low-dose methotrexate treatment. After the remission, maintenance therapy must be followed. The period of maintenance therapy varies widely. As long as there is no additional skin lesion found, the clinician has to taper the dosage of methotrexate while extending the interval for methotrexate therapy. According to a clinical study in Netherland, the follow-up visits for more than 5 years to check no additional lesion is very essential [6]. If the new lesion is developed, additional testing (histology, blood exam, and imaging) must be performed as required.

In conclusion, primary C-ALCL has more favorable prognosis than the other types of primary cutaneous lymphomas. However, as the clinicians who treat the skin wounds, we must carefully distinguish Primary cutaneous CD30+ LPD from the simple skin lesions with the ulceration and necrotic tissues. In a treatment plan, the surgical excision of the visible nodules or papules with the use of low-dose methotrexate can be a firstline treatment for multiple lesions of C-ALCL. 


\section{NOTES}

\section{Conflict of interest}

No potential conflict of interest relevant to this article was reported.

\section{Ethical approval}

The study was performed in accordance with the principles of the Declaration of Helsinki. Written informed consent was obtained.

\section{Patient consent}

The patient provided written informed consent for the publication and the use of his images.

\section{ORICD}

Bong Soo Baik https://orcid.org/0000-0002-8019-3310

Wu Seop Lee https://orcid.org/0000-0002-7540-4259

So Young Ji https://orcid.org/0000-0002-6060-1452

Ki Sung Park https://orcid.org/0000-0002-2672-2795

Wan Suk Yang https://orcid.org/0000-0002-2706-5678

Sun Young Kim https://orcid.org/0000-0001-9479-0549

\section{REFERENCES}

1. Willemze R, Jaffe ES, Burg G, Cerroni L, Berti E, Swerdlow SH, et al. WHO-EORTC classification for cutaneous lymphomas. Blood 2005; 105:3768-85.

2. Kempf W, Sander CA. Classification of cutaneous lymphomas: an update. Histopathology 2010;56:57-70.

3. Bradford PT, Devesa SS, Anderson WF, Toro JR. Cutaneous lymphoma incidence patterns in the United States: a population-based study of 3884 cases. Blood 2009;113:5064-73.
4. Sidiropoulos KG, Martinez-Escala ME, Yelamos O, Guitart J, Sidiropoulos M. Primary cutaneous T-cell lymphomas: a review. J Clin Pathol 2015;68:1003-10.

5. Lee HS, Suh KS, Lee DY, Cho KH, Oh SH, Kim SC, et al. Cutaneous lymphoma in Korea: a nationwide retrospective study. Acta Derm Venereol 2016;96:535-9.

6. Bekkenk MW, Geelen FA, van Voorst Vader PC, Heule F, Geerts ML, van Vloten WA, et al. Primary and secondary cutaneous CD30(+) lymphoproliferative disorders: a report from the Dutch Cutaneous Lymphoma Group on the long-term follow-up data of 219 patients and guidelines for diagnosis and treatment. Blood 2000;95:3653-61.

7. Strauss M, Kolkova Z, Laurian N, Zohar Y. Cutaneous malignant lymphoma of the nasal tip. Ann Otol Rhinol Laryngol 1986;95(2 Pt 1):208-10.

8. Fujita H, Nagatani T, Miyazawa M, Wada H, Koiwa K, Komatsu $\mathrm{H}$, et al. Primary cutaneous anaplastic large cell lymphoma successfully treated with low-dose oral methotrexate. Eur J Dermatol 2008;18:360-1.

9. Rodenhuis S, Kremer JM, Bertino JR. Increase of dihydrofolate reductase in peripheral blood lymphocytes of rheumatoid arthritis patients treated with low-dose oral methotrexate. Arthritis Rheum 1987;30:369-74.

10. Cooper JA Jr, White DA, Matthay RA. Drug-induced pulmonary disease. Part 1: cytotoxic drugs. Am Rev Respir Dis 1986; 133:321-40.

11. Searles G, McKendry RJ. Methotrexate pneumonitis in rheumatoid arthritis: potential risk factors. Four case reports and a review of the literature. J Rheumatol 1987;14:1164-71.

12. Weinblatt ME, Fraser P. Elevated mean corpuscular volume as a predictor of hematologic toxicity due to methotrexate therapy. Arthritis Rheum 1989;32:1592-6. 